\title{
Quantum Hall ferromagnets, cooperative transport anisotropy, and the random field Ising model
}

\author{
J. T. Chalker, ${ }^{1,2}$ D. G. Polyakov, ${ }^{3, *}$ F. Evers, ${ }^{3}$ A. D. Mirlin, ${ }^{1,3, \dagger}$ and P. Wölfle ${ }^{1,3}$ \\ ${ }^{1}$ Institut für Theorie der Kondensierten Materie, Universität Karlsruhe, 76128 Karlsruhe, Germany \\ ${ }^{2}$ Theoretical Physics, University of Oxford, 1 Keble Road, Oxford OX1 3NP, United Kingdom \\ ${ }^{3}$ Institut für Nanotechnologie, Forschungszentrum Karlsruhe, Karlsruhe 76021, Germany
}

(Received 2 August 2002; published 29 October 2002)

\begin{abstract}
We discuss the behavior of a quantum Hall system when two Landau levels with opposite spins and combined filling factor near unity are brought into energetic coincidence using an in-plane component of magnetic field. We focus on the interpretation of recent experiments under these conditions [Zeitler et al., Phys. Rev. Lett. 86, 866 (2001); Pan et al., Phys. Rev. B 64, 121305 (2001)], in which a large resistance anisotropy develops at low temperatures. Modeling the systems involved as Ising quantum Hall ferromagnets, we suggest that this transport anisotropy reflects domain formation induced by a random field arising from isotropic sample surface roughness.
\end{abstract}

DOI: 10.1103/PhysRevB.66.161317

PACS number(s): 73.43.Cd, 73.21.-b, 75.10.-b, 75.10.Nr

Two very striking experimental observations of large electronic transport anisotropy for quantum Hall systems in tilted magnetic fields have been reported recently. ${ }^{1,2}$ In both cases, anisotropy appears at integer values of the filling factor $\nu$, with an in-plane magnetic-field component tuned to bring two Landau levels of opposite spins into energetic coincidence. While the in-plane magnetic-field component itself defines an axis within the sample, the fact that large anisotropy appears in resistivity only below a characteristic temperature of about $1 \mathrm{~K}$ suggests that it has a cooperative origin. Our aim in this paper is to develop a theoretical treatment of such systems and to discuss the source of the observed anisotropy.

In view of the phenomenological similarities, it is natural to make comparisons between these Landau-level coincidence experiments and the earlier discovery of resistance anisotropy in quantum Hall systems near half filling of high Landau levels, ${ }^{3}$ attributed to the formation of a uniaxial charge-density wave with a period set by the cyclotron radius. ${ }^{4}$ Some distinctions are, however, clear. Most importantly, the nature of the electron states near the chemical potential and their average occupation is quite different in each case: two separate orbital Landau levels with opposite spins and a combined filling factor close to unity are involved in the coincidence experiments, as against a single, spin-polarized and roughly half filled Landau level in the other case. In this context it is desirable to examine a range of possible explanations for low-temperature anisotropy.

The study of cooperative effects in coincident Landau levels has a long history. Consider a system with fixed magnetic-field strength $B_{\perp}$ perpendicular to the twodimensional electron or hole gas, as a function of total field strength $B_{\text {tot }}$. In a single-particle description, there are pairs of Landau levels having opposite spin orientations and orbital quantum numbers differing by $\Delta N$, which are separated in energy by $\Delta N \hbar \omega_{c}-g^{*} \mu_{\mathrm{B}} B_{\text {tot }}$, where $\omega_{c} \propto B_{\perp}$ is the cyclotron frequency and $g^{*} \mu_{\mathrm{B}} B_{\text {tot }}$ is the Zeeman contribution. This energy gap falls to zero at coincidence. Inclusion of exchange interactions leads, at a combined filling factor of unity for the crossing levels, to a first-order transition between two ground states in which one or other level is com- pletely filled. Within a Hartree-Fock treatment, the excitation gap remains nonzero through this transition. ${ }^{5}$ Early observations of a quantized plateau in Hall resistivity $\rho_{x y}$ and deep minimum in diagonal resistivity $\rho_{x x}$, both persisting through the transition, ${ }^{6}$ provide support for such a picture, while measurements of the temperature dependence of $\rho_{x x}$ allow the determination of an activation energy gap ${ }^{7}$ which has the variation with $B_{\|}$expected theoretically. This Landau-level coincidence transition is one example of a broad class of cooperative phenomena in quantum Hall systems, involving ferromagnetism of either spin or pseudospin variables, which have been a focus for much recent work. ${ }^{8,9}$ From such a perspective, representing the two Landau levels involved using two states of a pseudospin, interactions between pseudospins are ferromagnetic with Ising anisotropy, ${ }^{10}$ while $B_{\text {tot }}$ measured from its value at coincidence acts on pseudospins as a Zeeman field.

With this background in mind, we return to a discussion of transport anisotropy. Following the suggestions of Refs. 1 and 2, it is clear that the presence of a spin or charge density wave could potentially explain this observation. From the original Hartree-Fock calculations, ${ }^{5}$ which were for a oneband model and, considered only trial states with homogeneous charge density, it was found that instability to a spindensity wave is preempted by the first-order coincidence transition. An escape from this conclusion might be provided by the fact that the sample involved in one experiment is a $\mathrm{Si} / \mathrm{GeSi}$ heterostructure ${ }^{1}$ and in the other a wide quantum well ${ }^{2}$ the former has valley degeneracy and the latter has two occupied subbands. Alternatively, it might be that a better trial state in the Hartree-Fock theory, or calculations that go beyond this approximation, would yield a stripe phase as the true ground state near coincidence. However, recent Hartree-Fock calculations for bilayer systems ${ }^{11}$ give only ferromagnetic pseudospin order with parameters relevant in the present context, as do calculations for one-band models, in which the Hartree-Fock solutions with both spin- and chargedensity modulations are considered, ${ }^{12}$ or the Hamiltonian with realistic interaction potentials is diagonalized exactly for a small number of electrons. ${ }^{13}$ 
The observations of Refs. 1 and 2 therefore present a puzzle, which, we argue in the following, can be understood in terms of domain formation, with a characteristic size much larger than the relevant scale for stripe phases, the cyclotron radius. Our account involves three distinct ingredients. First, we suggest that domains are induced by a random Zeeman field acting on the pseudospins, which arises from the interplay between isotropic sample surface roughness and the in-plane component of magnetic field. Second, we show that a random field generated by this mechanism is intrinsically endowed with anisotropic correlations, and that the correlation anisotropy is large enough to explain the observed anisotropy in resistivity. Third, we argue that transport in a multidomain sample occurs along domains walls via the processes discussed recently in Refs. 14-16. The onset temperature for transport anisotropy arising by this mechanism is the Curie temperature of the Ising quantum Hall ferromagnet, and we note that the reported ${ }^{1,2}$ onset temperature of about 1 $\mathrm{K}$ is similar to the value for the Curie temperature expected ${ }^{10}$ and the value observed elsewhere. ${ }^{17}$

As a starting point for a more detailed discussion, consider an energy functional for the system. Introducing coherent-state creation operators $c_{\uparrow}^{\dagger}(\mathbf{r})$ and $c_{\downarrow}^{\dagger}(\mathbf{r})$ for the two Landau levels involved, correlations are characterized by the expectation value of pseudospin, $\mathbf{S}(\mathbf{r})=\left\langle c_{\alpha}^{\dagger} \boldsymbol{\sigma}_{\alpha \beta} c_{\beta}\right\rangle$, where $\boldsymbol{\sigma}$ is the vector of Pauli matrices. The order parameter has magnitude $|\mathbf{S}(\mathbf{r})|=S$, where $S=1$ at a combined filling factor of unity for the two Landau levels and is smaller otherwise. For variations of $\mathbf{S}(\mathbf{r})$, which are smooth on the scale of the cyclotron radius, one expects the energy functional to have the form

$$
E=\int\left(-D S_{z}^{2}+J|\nabla \mathbf{S}|^{2}+\delta J\left|\partial_{n} \mathbf{S}(\mathbf{r})\right|^{2}-h S_{z}\right) d^{2} \mathbf{r} .
$$

Here $D>0$ represents Ising anisotropy, $J$ is the spin stiffness, the derivative $\partial_{n} \equiv \hat{\mathbf{n}} \cdot \boldsymbol{\nabla}$ acts in the direction of the in-plane magnetic field, denoted by $\hat{\mathbf{n}}$, and $\delta J$ represents spatial anisotropy in the spin stiffness (for simplicity, we omit anisotropy in spin space from the stiffness). The effective Zeeman field acting on pseudospins is $h$. In experiment, the strength of this field varies through zero as the tilt angle $\theta$ of the sample in the applied magnetic field is varied through the Landau-level coincidence point; its strength depends also on field magnitude $B_{\text {tot }}$ and on carrier density $n$.

For a homogeneous system, the ground state of Eq. (1) is uniform with $S_{z}=\operatorname{sgn}(h) S$ and $\mathbf{S}_{\perp}=\mathbf{0}$. Domains may arise either in metastable states or because they are induced by quenched disorder. While metastability and hysteresis are observed in some examples of quantum Hall ferromagnets, ${ }^{17}$ this is not reported to be an important aspect of observations in Refs. 1 and 2. We therefore turn to domains induced by disorder. Potentially, the most important source of disorder in Eq. (1) is randomness in $h$, and the behavior of the randomfield Ising model has been studied very extensively. ${ }^{18}$ It is useful to distinguish the weak and strong disorder regimes: taking $h$ to fluctuate about mean value zero with amplitude $\Delta$ and correlation length $l$, and supposing $l$ to be greater than the domain-wall width $w=\sqrt{J / D}$, the boundary between the two regimes lies at $l \Delta \sim \sqrt{J D}$. At weak disorder, domain size $\xi$ is much larger than $l$ and domain morphology depends, amongst other things, on the difference in energy per unit length of domain walls running parallel or perpendicular to the in-plane magnetic-field component. This energy difference is of the order $\sqrt{\delta J D}$. At strong disorder, which we shall argue is the limit relevant here, the domain pattern is simply that of $\operatorname{sgn}(h)$.

To apply these ideas, it is necessary to identify a microscopic origin for such a random field. One possibility is that variations in carrier density $n$, arising either from impurity scattering or from large-scale inhomogeneities, produce changes in the value of $h$. Randomness of this kind is spatially isotropic, but may give rise to transport anisotropy via dependence of the domain-wall energy on spatial orientation. A second possibility is that sample surface roughness changes the local value of $\theta$, and hence $h$. To compare the likely importance of these two, we appeal to experiment, noting (for example, from Fig. 2 of Ref. 1) that while the coincidence transition has a rather small width $\left(0.5^{\circ}\right)$ in $\theta$, it has a much larger width $(20 \%)$ in $B_{\text {tot }}$, which is indicative of its width in $n$. The existence of sample surface roughness with an amplitude of a few nonometers and $l \sim 1 \mu \mathrm{m}$ is reported in Ref. 1 and amplitudes of up to $10 \mathrm{~nm}$ are well established in a variety of other contexts, ${ }^{19}$ giving gradients of at least a few tenths of a degree. We estimate that the condition $l \Delta \sim \sqrt{J D}$ is met by surface height fluctuations of order $\cot \left(\theta_{c}\right) e^{2} / \epsilon \hbar \omega_{c}$, and conclude that the random field originating from surface roughness constitutes intermediate or strong disorder. Moreover, domain formation by this mechanism can account for transport anisotropy, as we now show.

Let $z(\mathbf{r})$ denote height of the sample surface above an average reference plane, and let $\theta_{c}$ be the critical angle at which the Landau-level coincidence transition occurs. Then for small-angle roughness,

$$
h(\mathbf{r})=\alpha\left(\theta-\theta_{c}\right)+\alpha \partial_{n} z(\mathbf{r}),
$$

where $\alpha$ is a proportionality constant. Crucially, by this mechanism, surface roughness with a correlation function

$$
C(\mathbf{r})=\left\langle z\left(\mathbf{r}^{\prime}\right) z\left(\mathbf{r}+\mathbf{r}^{\prime}\right)\right\rangle-\left\langle z\left(\mathbf{r}^{\prime}\right)\right\rangle\left\langle z\left(\mathbf{r}+\mathbf{r}^{\prime}\right)\right\rangle,
$$

which is isotropic, generates a random field with spatially anisotropic correlations, since

$$
\left\langle h\left(\mathbf{r}^{\prime}\right) h\left(\mathbf{r}+\mathbf{r}^{\prime}\right)\right\rangle-\left\langle h\left(\mathbf{r}^{\prime}\right)\right\rangle\left\langle h\left(\mathbf{r}+\mathbf{r}^{\prime}\right)\right\rangle=-\alpha^{2} \partial_{n}^{2} C(\mathbf{r}) .
$$

To establish the characteristic degree of this anisotropy, we have carried out numerical simulations. Taking $z(\mathbf{r})$ to be a superposition of overlapping Gaussian functions of position, with centers placed at random points in the plane and amplitudes distributed uniformly about zero, and setting $\theta$ $=\theta_{c}$, the resulting random field $h(\mathbf{r})$ in a typical realization is illustrated in Fig. 1. Anticipating our discussion of transport on domain walls, we quantify the anisotropy evident in this figure by following the classical dynamics of a particle that moves along contours of $h(\mathbf{r})$, using methods described previously. $^{20}$ Averaging over randomly placed starting 

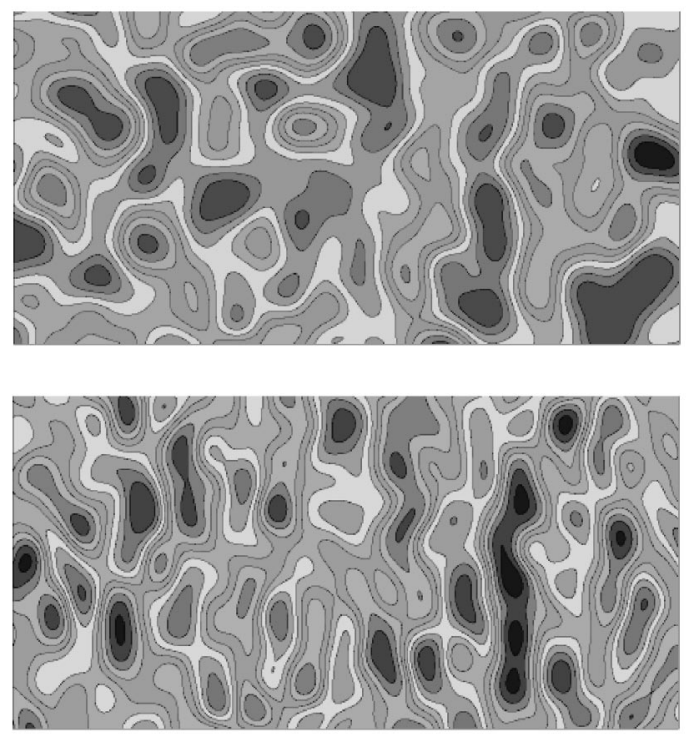

FIG. 1. Gray scale plots of (upper panel) spatially isotropic surface roughness $z(\mathbf{r})$ and (lower panel) the spatially anisotropic Ising-model random field $\partial_{n} z(\mathbf{r})$ that results from this surface roughness.

points, we expect diffusive motion in the sense that meansquare displacements grow linearly in time. Taking $\hat{\mathbf{n}}$ parallel to the $y$ axis, the quantities $D_{x x}(t) \equiv\left\langle x^{2}(t)\right\rangle / t$ and $D_{y y}(t)$ $\equiv\left\langle y^{2}(t)\right\rangle / t$ should then approach the eigenvalues $D_{x x}$ and $D_{y y}$ of the diffusion tensor, for times $t$ that are large compared to the correlation time $t_{0}$. Evidence that $D_{x x}(t)$ and $D_{y y}(t)$ indeed tend to a finite limit, with $D_{x x} \sim 8 D_{y y}$, is presented in Fig. 2. The orientation of this anisotropy, with the larger diffusion constant in the direction perpendicular to the in-plane magnetic-field component, is as observed in Refs. 1,2 , and its magnitude is about the same as that determined at low temperature using a Hall bar sample. ${ }^{1}$ The precise value of $D_{x x} / D_{y y}$ will be dependent on the disorder distribution, but we see no reason to expect large variations. Our calculations also provide an opportunity to test the universality class of our anisotropic percolation problem, since diffusive

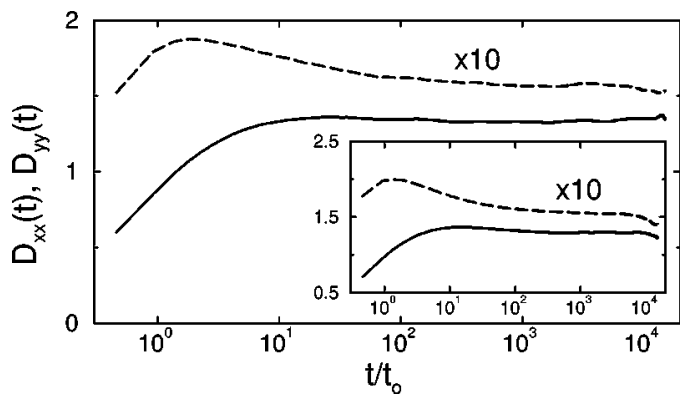

FIG. 2. Simulation data used to determine diffusion coefficient anisotropy. Mean-square displacements per unit time in directions perpendicular $\left[D_{x x}(t)\right.$, full line $]$ and parallel $\left[D_{y y}(t)\right.$, dashed line $]$ to the in-plane field direction $\hat{\mathbf{n}}$ are shown, averaged over all trajectories. Inset shows the averages over open trajectories only, $\left\langle x^{2}(t)\right\rangle / t^{8 / 7}$ and $\left\langle y^{2}(t)\right\rangle / t^{8 / 7}$, demonstrating scaling with the classical percolation exponent value.
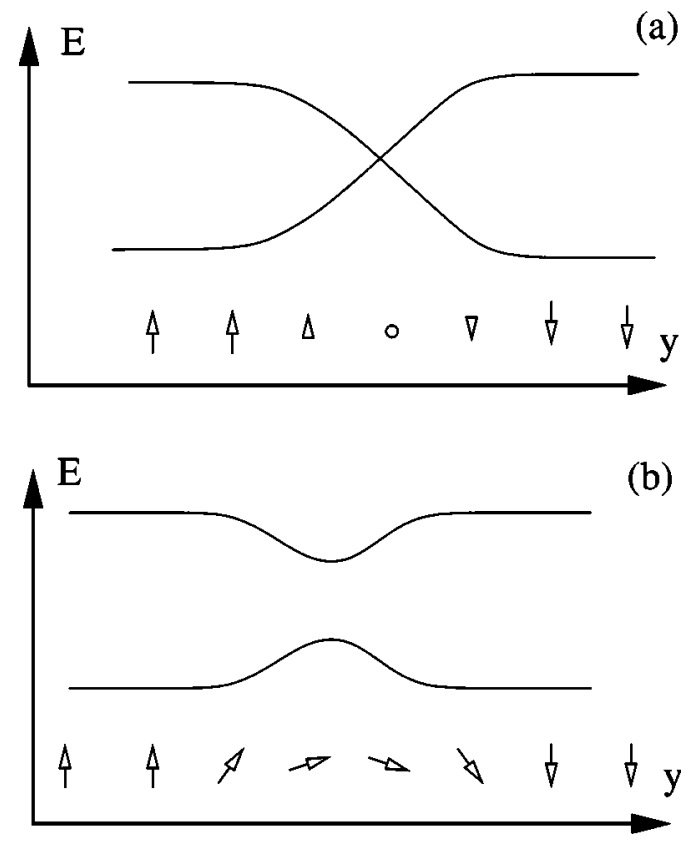

FIG. 3. Schematic summary of domain-wall structure, showing pseudospin and excitation energies $E$, as a function of the position $y$ across the wall, within the Hartree-Fock theory; (a) for an Ising wall stabilized by short-range scattering, and (b) for a Bloch wall.

growth in the mean-square displacement arises at long times from a balance between bounded motion on closed trajectories and super diffusive motion on trajectories that remain open up to the observation time. ${ }^{21}$ Averaging only over open trajectories, one expects $\left\langle x^{2}(t)\right\rangle \propto\left\langle y^{2}(t)\right\rangle \propto t^{8 / 7}$, if the anisotropic problem is in the same universality class as the standard classical percolation problem; data shown in the inset of Fig. 2 support this conclusion.

The foregoing discussion is based on the idea that transport occurs along boundaries between domains. In order to substantiate this, we next examine transport properties of domain walls between oppositely magnetized phases of the Ising quantum Hall ferromagnet. Recalling that the domain wall forms the boundary between a region on one side with filling factors for the coincident Landau levels of $\nu_{\uparrow} \simeq 1$ and $\nu_{\downarrow} \simeq 0$, and a region on the other with interchanged filling factors, $\nu_{\uparrow} \simeq 0$ and $\nu_{\downarrow} \simeq 1$, the simplest structure one might imagine is that shown in Fig. 3(a). In this picture, the wall supports two counterpropagating modes with opposite spin polarizations, which arise as edge states of the occupied Landau levels in the domains on either side. Such an Ising domain wall, in which $\mathbf{S}_{\perp}(\mathbf{r})=0$ everywhere and $\mathbf{S}(\mathbf{r})=0$ at the wall center, may be stabilized by short-range scattering, which allows solutions with $|\mathbf{S}(\mathbf{r})|<1,{ }^{22}$ in contrast to Eq. (1). For a sample without short-range scattering, however, Hartree-Fock theory yields ${ }^{16}$ the Bloch domain-wall structure shown in Fig. 3(b). Here, $\mathbf{S}_{\perp}(\mathbf{r}) \neq 0$ on the wall. Consequently, within Hartree-Fock theory there is mixing and an avoided crossing of edge states arising from occupied Landau levels on either side of the wall.

At this level of approximation, for a Bloch wall the chemical potential lies within a quasiparticle gap. To account for transport under these conditions, it is necessary to con- 
sider collective excitations. The combined consequences of continuous symmetry for the Hartree-Fock solution under rotations of $\left\langle\mathbf{S}_{\perp}(\mathbf{r})\right\rangle$ about the Ising axis, and the connection between spin or pseudospin and charge that is standard for quantum Hall ferromagnets, ${ }^{8}$ have been examined in a related context in Ref. 15. Introducing pseudospin rotation angle $\varphi$ as a function of position coordinate $x$ along the wall and imaginary time $\tau$, the action

$$
S=\frac{\rho}{2} \iint\left[\left(\frac{\partial \varphi}{\partial x}\right)^{2}+\frac{1}{u^{2}}\left(\frac{\partial \varphi}{\partial \tau}\right)^{2}\right] d x d \tau
$$

is obtained for domain-wall excitations, ${ }^{15}$ where in our context $\rho \sim J w \sim e^{2} / \epsilon$ and $u \sim e^{2} / \epsilon \hbar$. A charge density $(2 \pi)^{-1} \partial \varphi / \partial x$ is associated with these modes. This is the action for a spinless Luttinger liquid. A vital property for our argument is that left- and right-moving excitations propagate independently, provided rotation symmetry about the pseudospin easy axis is exact. In short, Fig. 3(a) remains a useful picture even without the short-range disorder to stabilize an Ising wall, provided only that there is no spin-orbit scatter- ing. In this picture, transport in a multidomain sample occurs at domain boundaries, via two independent, counterpropagating sets of modes. Neglecting quantum interference effects, we arrive at the problem for which numerical results are given above.

In conclusion, we have argued that the observations of anisotropic transport reported in Refs. 1 and 2 can plausibly be attributed to the formation of anisotropically shaped domains, induced as a result of sample surface roughness. Our numerical work demonstrates that this mechanism generates an anisotropy comparable to that found experimentally. ${ }^{1}$ In addition, the onset temperature for strongly anisotropic transport is comparable to the critical temperature expected ${ }^{10}$ and observed $^{17}$ in other Ising quantum Hall ferromagnets. For future work, it would be interesting to investigate transport in systems with deliberately induced surface features.

We thank U. Zeitler for extensive discussions and E. H. Rezayi for correspondence. The work was supported by the A. v. Humboldt Foundation (J.T.C.), by the Russian Fund for Basic Research, and the Schwerpunktprogramm "QuantenHall-Systeme" der Deutschen Forschungsgemeinschaft.
*Also at A. F. Ioffe Physico-Technical Institute, 194021 St. Petersburg, Russia.

${ }^{\dagger}$ Also at Petersburg Nuclear Physics Institute, 188350 St. Petersburg, Russia.

${ }^{1}$ U. Zeitler et al., Phys. Rev. Lett. 86, 866 (2001).

${ }^{2}$ W. Pan et al., Phys. Rev. B 64, 121305 (2001).

${ }^{3}$ M.P. Lilly et al., Phys. Rev. Lett. 82, 394 (1999); R.R. Du et al., Solid State Commun. 109, 389 (1999).

${ }^{4}$ A.A. Koulakov, M.M. Fogler, and B.I. Shklovskii, Phys. Rev. Lett. 76, 499 (1996); M.M. Fogler, A.A. Koulakov, and B.I. Shklovskii, Phys. Rev. B 54, 1853 (1996); R. Moessner and J.T. Chalker, ibid. 54, 5006 (1996).

${ }^{5}$ G.F. Giuliani and J.J. Quinn, Phys. Rev. B 31, 6228 (1985).

${ }^{6}$ S. Koch et al., Phys. Rev. B 47, 4048 (1993).

${ }^{7}$ A.J. Daneshvar et al., Phys. Rev. Lett. 79, 4449 (1997).

${ }^{8}$ S.L. Sondhi et al., Phys. Rev. B 47, 16419 (1993).

${ }^{9}$ For an introduction, see S.M. Girvin, in Ecole des Houches: Topological Aspects of Low Dimensional Systems, edited by A. Comtet, T. Jolicouer, and S. Ouvry (Les Editions de Physique, Paris, 1999).
${ }^{10}$ T. Jungwirth et al., Phys. Rev. Lett. 81, 2328 (1998); T. Jungwirth and A.H. MacDonald, Phys. Rev. B 63, 035305 (2000).

${ }^{11}$ E. Demler et al., cond-mat/0110126.

${ }^{12}$ F. Evers and D. G. Polyakov (unpublished).

${ }^{13}$ T. Jungwirth, A.H. MacDonald, and E.H. Rezayi (unpublished).

${ }^{14}$ V.I. Falko and S.V. Iordanskii, Phys. Rev. Lett. 82, 402 (1999).

${ }^{15}$ A. Mitra and S.M. Girvin, cond-mat/0110078 (unpublished).

${ }^{16}$ L. Brey and C. Tejedor, Phys. Rev. B 66, 041308 (2002).

${ }^{17}$ V. Piazza et al., Nature (London) 402, 638 (1999).

${ }^{18}$ Y. Imry and S.-K. Ma, Phys. Rev. Lett. 35, 1399 (1975); for a review, see Spin Glasses and Random Fields, edited by A.P. Young (World Scientific, Singapore, 1997).

${ }^{19}$ C. Orme et al., Appl. Phys. Lett. 64, 860 (1994); R.L. Willett et al., Phys. Rev. Lett. 87, 126803 (2001); K.B. Cooper et al., Solid State Commun. 119, 89 (2001).

${ }^{20}$ F. Evers and W. Brenig, Z. Phys. B: Condens. Matter 94, 115 (1994).

${ }^{21}$ R.M. Ziff, X.P. Kong and E.G.D. Cohen, Phys. Rev. A 44, 2410 (1991).

${ }^{22}$ S. Yarlagadda, Phys. Rev. B 44, 13101 (1991). 